\section{Evaluación cuantitativa de la maduración ósea en vértebras cervicales con el uso de la tomografía computarizada Cone Beam}

\section{Quantitative cervical vertebral maturation assessment with the use of Cone-Beam computed tomography}

\begin{abstract}
Resumen
Objetivo: Evaluar un método cuantitativo de maduración ósea en vértebras cervicales en 14 tomografías computarizadas Cone Beam de niños de 9 a 15 años de edad obtenidas del archivo de la Clínica de Imagenología de la Facultad de Odontología de la Universidad Nacional Mayor San Marcos. Métodos: Se realizó un estudio retrospectivo, descriptivo y transversal. La muestra fue por conveniencia de 14 tomografías de niños (siete del sexo masculino), éstas fueron imágenes clínicamente justificadas, tomadas previamente con el equipo Picasso Master 3D - FOV 20x15 y procesadas con el software EzImplant; se posicionó la cabeza teniendo como referencia el plano de Frankfort y se realizaron los cortes. Se empleó un método cuantitativo que estableció cuatro estadios de maduración de manera objetiva mediante una ecuación que empleó tres mediciones en las vértebras C2, C3 y C4. Resultados: El mayor porcentaje de niños se encontró en el periodo de desaceleración. En el periodo de alta velocidad, se halló los valores más altos para el sexo femenino con una edad promedio de 11 años. Conclusiones: El método cuantitativo descrito fue sencillo, práctico y aplicable a través de la tomografía computarizada Cone Beam mostrando buenos resultados.
\end{abstract}

Palabras clave: Evaluación; Desarrollo óseo; Vértebras cervicales; Tomografía computarizada por rayos $\mathrm{X}$.

\begin{abstract}
Objective: To evaluate a quantitative method of bone maturation in cervical vertebrae in 14 Cone Beam computed tomographies of children aged 9 to 15 years old obtained from the archive of the Radiologic Service of the School of Dentistry at the National University of San Marcos. Methods: A retrospective, descriptive and transversal study was carried out. The sample was for convenience of 14 tomographies of children (seven male), these were clinically justified images, previously taken with the Picasso Master 3D - FOV 20x15 and processed with the EzImplant software; the head was positioned with reference to the Frankfort plane and the cuts were made. We used a quantitative method that established four stages of maturation in an objective way by means of an equation that used three measurements in the vertebrae C2, C3 and C4. Results: The highest percentage of children was found in the deceleration period. In the high-speed period, the highest values were found for the female sex with an average age of 11 years. Conclusions: The quantitative method described was simple, practical and applicable through Cone Beam computed tomography showing good results.
\end{abstract}

Keywords: Evaluation; Bone development; Cervical vertebrae; Tomography X-Ray computed

\section{Artículo Original}

Rocío del Pilar Ríos León ${ }^{1, a}$, Jessica Salas Huallparimache ${ }^{1, a}$, Cynthia Salazar Zapata ${ }^{1, a}$ Sue Salas Catacora ${ }^{1, a}$, Gina Flores Díaz,a, Carlos Tisnado Florián ${ }^{1, b}$, Daniel Blanco Victorio $1,2, c$

${ }^{1}$ Facultad de Odontología. Universidad Nacional Mayor de San Marcos. Lima, Perú.

${ }^{2}$ Facultad de Ciencias. Universidad Peruana Cayetano Heredia. Lima, Perú.

${ }^{a}$ Cirujano Dentista.

${ }^{\mathrm{b}}$ Especialista en Ortodoncia y Ortopedia Maxilar.

c Especialista en Estadística en Investigación.

Correspondencia:

Rocío del Pilar Ríos León

Correo electrónico: rocioriosl@hotmail.com $\mathrm{Mz}$ B Lote 35 . Urb Santo Domingo $1^{\circ}$ etapa Carabayllo. Lima. Perú.

Coautores:

Jessica Salas Huallparimache

jessisal24@hotmail.com

Cynthia Salazar Zapata

cinthyasalazarzapata@gmail.com

Sue Salas Catacora

sue.essc@hotmail.com

Gina Flores Díaz

judiamiga@hotmail.com

Carlos Tisnado Florián

itisnado@hotmail.com

Daniel Blanco Victorio

danielblanco92@outlook.com

Conflicto de intereses: Los autores declaran no tener conflictos de interés.

Fuente de financiamiento: Autofinanciado

Fecha de recepción: 11/12/17

Fecha de aceptación: 27/02/18

( ) Los autores. Este artículo es publicado por la revista Odontología Sanmarquina de la Facultad de Odontología, Universidad Nacional Mayor de San Marcos. Este es un artículo de acceso abierto, distribuido bajo los términos de la licencia Creative Commons Atribucion - No Comercia_Compartir Igual 4.0 Internacional. (http://creativecommons.org/licenses/by-nc-sa/4.0/) que permite el uso no comercial, distribución y reproducción en cualquier medio, siempre que la obra original sea debidamente citada. 


\section{Introducción}

Los métodos de maduración ósea nos ayudan a identificar los períodos de crecimiento del individuo. Es importante determinar en qué período intervenir a los pacientes para el diagnóstico, evaluación y planeación del tratamiento ortodóncico $\mathrm{u}$ ortopédico puesto que se observa una mejor respuesta al tratamiento si se realiza cuando se alcanza el período de aceleración o punto máximo de crecimiento puberal; en este momento tanto la mandíbula como el resto del cuerpo alcanzan su mayor grado de evolución, de esa forma podemos corregir los desbalances esqueléticos ${ }^{1-4}$. Cabe destacar que la maduración ósea es un proceso continuo y que el crecimiento es una variable crítica en Ortodoncia influenciada por múltiples factores, entre ellos: genética, etnia, nutrición y nivel socioeconómico ${ }^{5}$.

Entre los Métodos de Maduración Ósea se encuentra el Índice de Maduración Esqueletal (SMI, por sus siglas en inglés) propuesto por Fishman ${ }^{6}$ en la cual se utiliza la radiografía de mano-muñeca, siendo considerada en la actualidad aún como el gold standard en investigaciones y la práctica clínica ${ }^{7}$.Otro método es la evaluación de la Maduración Ósea en Vértebras Cervicales (CVM, por sus siglas en inglés) en cefalogramas y puede ser un reemplazo a la de mano-muñeca ya que no necesita una radiografía adicional ${ }^{2,8}$. Fue originalmente desarrollada por Lamparski ${ }^{9}$ y sucesivamente mejorada por O'Reilly ${ }^{10}$, Hassel y Farman ${ }^{11}$ y Baccetti et al. ${ }^{12}$, y está basada en criterios cualitativos donde se evalúa la forma de las vértebras, especialmente la C2, C3 y C4, para determinar la edad ósea ${ }^{13,14}$. Aunque este método ha sido validado, algunos estudios han cuestionado su aplicabilidad como Chatzigianni y Halazonetis ${ }^{15}$ y Gabriel et al. ${ }^{16}$ los cuales han reportado la pobre reproducibilidad de estos métodos tanto intra e interexaminador. Es por ello que diversos investigadores proponen un nuevo método cuantitativo para estimar de forma objetiva la maduración ósea y reducir la variabilidad de las interpretaciones ${ }^{17-20}$. En ese sentido, Mito et al. ${ }^{17}$ establecieron la edad ósea en vértebras cervicales de forma cuantitativa (QCVM, por sus siglas en inglés) en radiografías cefalométricas y reflejaron la madurez esqueletal al igual que la de mano-muñeca. En el 2008, Chen et al. ${ }^{21}$ tuvieron el objetivo de establecer un sistema cuantitativo de CVM en cefalogramas, donde se seleccionaron tres mediciones de 42 de las vértebras C2, C3 y C4, mediante una correlación con el método SMI de mano - muñeca (Fishman) para desarrollar una ecuación y así determinar cuatro etapas de maduración ósea; concluyendo que su método es objetivo para evaluar la maduración ósea. Santiago et al. ${ }^{19}$ en el 2014 desarrollaron y validaron un software para un análisis cuantitativo de la maduración ósea en vértebras cervicales en cefalogramas. Así como, Byun et al. ${ }^{20}$ estimaron las etapas de maduración ósea con parámetros de CVM (el proceso odontoide y el cuerpo de la C2, C3 y C4) generados por la Tomografía Computarizada Cone Beam (CBCT, por sus siglas en inglés) mediante modelos de regresión múltiple, es decir una evaluación cuantitativa. Gray et $a l^{22}$, en un estudio longitudinal realizaron un análisis morfométrico de la C2, C3 y C4 en radiografías cefalométricas, (es decir un análisis cuantitativo) en 25 individuos en crecimiento y estos cambios fueron consistentes con la descripción del CVM pero a diferencia de estudios anteriores, estos cambios no pudieron identificar con precisión el pico de crecimiento mandibular.

En el presente trabajo se empleó la CBCT ya que es una herramienta de diagnóstico y planificación en casos específicos de Ortodoncia ${ }^{14,23}$ que brinda imágenes tridimensionales con beneficios potenciales ya que permite obtener mediciones exactas de las vértebras ${ }^{20}$. De los métodos revisados se optó por el propuesto por Chen et al. ${ }^{21}$ ya que es sencillo, práctico y objetivo. Se debe señalar que existe poca información en Latinoamérica sobre el uso de un método cuantitativo de la maduración ósea, es por ello que nuestro objetivo fue evaluar el método de maduración ósea en vértebras cervicales propuesto por Chen et al. ${ }^{21}$ (QCVM), empleando esta vez la CBCT para que de una manera precisa se pueda obtener los estadios de maduración ósea de los pacientes.

\section{Métodos}

Se diseñó un estudio piloto, retrospectivo, descriptivo y transversal con una muestra por conveniencia de 14 tomografías de niños ( 7 del sexo masculino y 7 del sexo femenino) de 9 a 15 ańos de edad. Dichas tomografías, clínicamente justificadas, fueron obtenidas del archivo de la clínica de Imagenología de la Facultad de Odontología de la Universidad Nacional Mayor de San Marcos. Las imágenes por CBCT no fueron tomadas específicamente para el presente estudio, sino que fueron obtenidas previamente a solicitud de una especialidad odontológica (Ortodoncia y/o Cirugía). Se excluyeron las tomografías de los niños con alguna malformación congénita que afecten la curvatura normal de la columna vertebral o que las imágenes de las tomografías de las vértebras C2, C3 y C4 carecieran de nitidez. Las tomografías fueron tomadas con un equipo Picasso Master 3D (Vatech, Hwaseong, South Korea, $8 \mathrm{Ma}, 90 \mathrm{kpv}$ ) con un campo de visión (FOV) de $20 \mathrm{~cm} \times 15 \mathrm{~cm}$.

Las imágenes fueron procesadas con el software EzImplant. Una vez dentro del software y antes de comenzar el análisis, se posicionó la cabeza del paciente teniendo como referencia el plano de Frankfurt según Swennen ${ }^{24}$ (Figura 1); para realizar los cortes se ubicó la parte más posterior del foramen de la segunda vértebra cervical y luego el centro del cuerpo, generando el eje anteroposterior en vista axial (Figura 2A). Después de obtenido este eje se ajustó al eje vertical para pasarlo por el centro del proceso odontoides en una vista coronal (Figura 2B). La imagen sagital de los cuerpos vertebrales de C2 a C4 se obtuvo del plano formado por el eje vertical y el eje anteroposterior. Con un examinador previamente capacitado se procedió a realizar las mediciones en base 


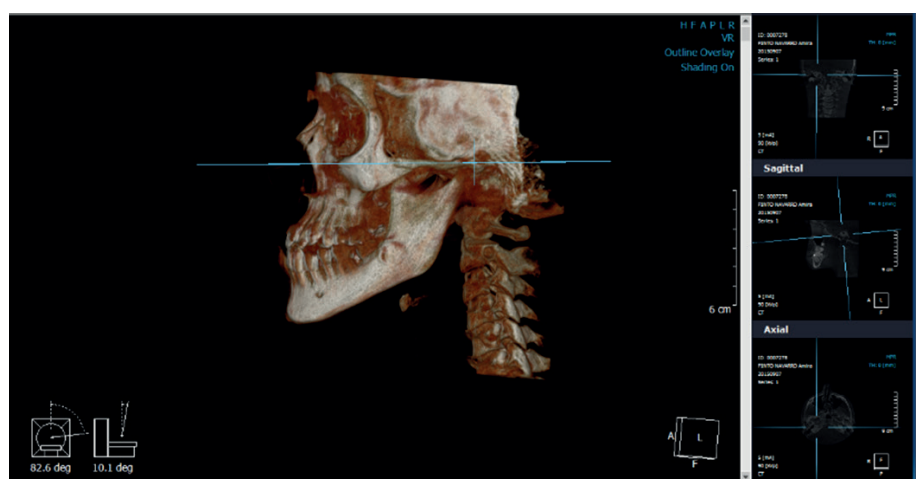

Figura 1. Posición de la cabeza paralela al plano de Frankfurt en vista 3D en CBCT
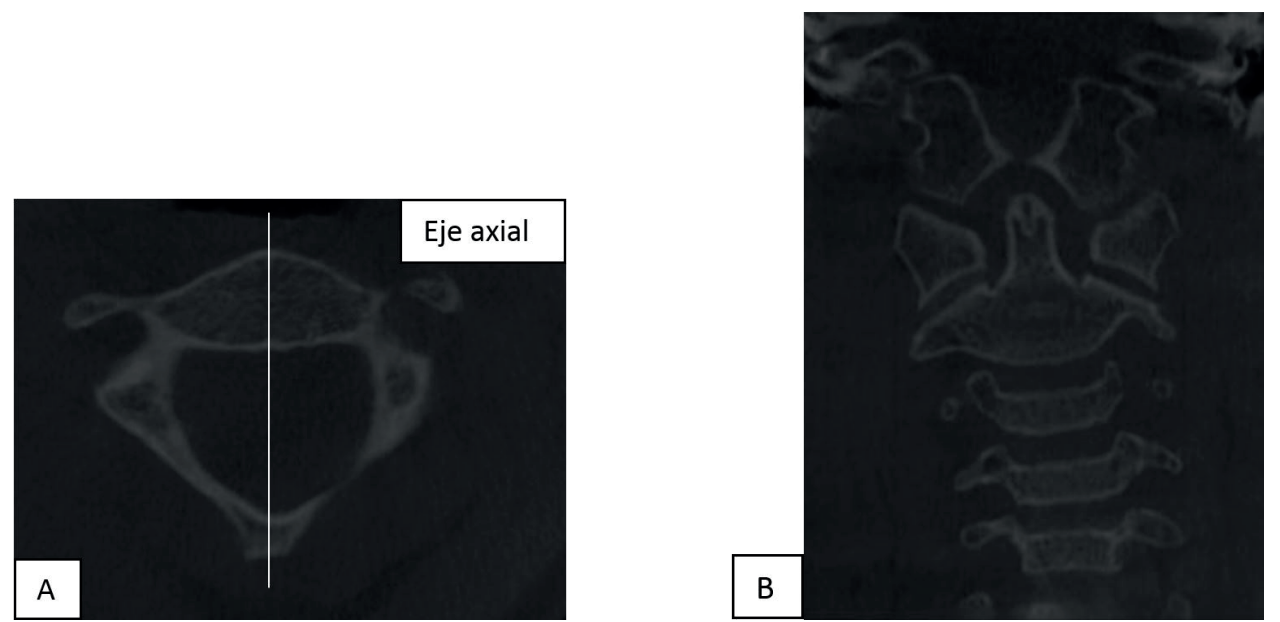

Figura 2. Imágenes de la tomografía Cone Beam mostrando la vértebra C2. A. Imagen axial, ubicación de la parte más posterior del foramen de la C2 y el centro del cuerpo de la C2 generando el eje anteroposterior. B. Imagen frontal, después de obtenido el eje anteroposterior se ajustó al eje vertical para pasarlo por el centro del proceso odontoides

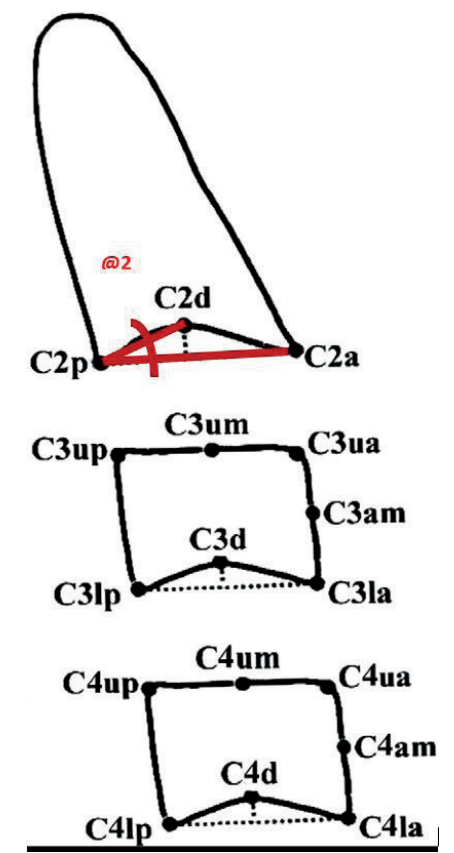

Figura 3. Puntos usados en el análisis de los cuerpos vertebrales. @2: ángulo anterosuperior de C2d a C2p con la proyección de $\mathrm{C} 2 \mathrm{p}$ a $\mathrm{C} 2 \mathrm{a}$. $\mathrm{C} 2 \mathrm{~d}, \mathrm{C} 3 \mathrm{~d}$ y $\mathrm{C} 4 \mathrm{~d}$, el punto más superior del borde inferior de los cuerpos de la $\mathrm{C} 2$, C3, y C4, respectivamente. C2a, C2p, C3la, C3lp, C4la, y C4lp, los puntos más anteriores y más posteriores del borde inferior de los cuerpos de la C2, C3, y C4 respectivamente. C3ua, C3up, C4ua, y C4up, los puntos más superiores de los bordes anteriores y posteriores de los cuerpos de la C3 y C4. C3um y C4um, punto medio del borde superior de los cuerpos de la C3 y C4. C3am y C4am, punto medio del borde anterior de los cuerpos de la C3 y C4. Fuente: Obtenido de Chen et al. ${ }^{21}$ 

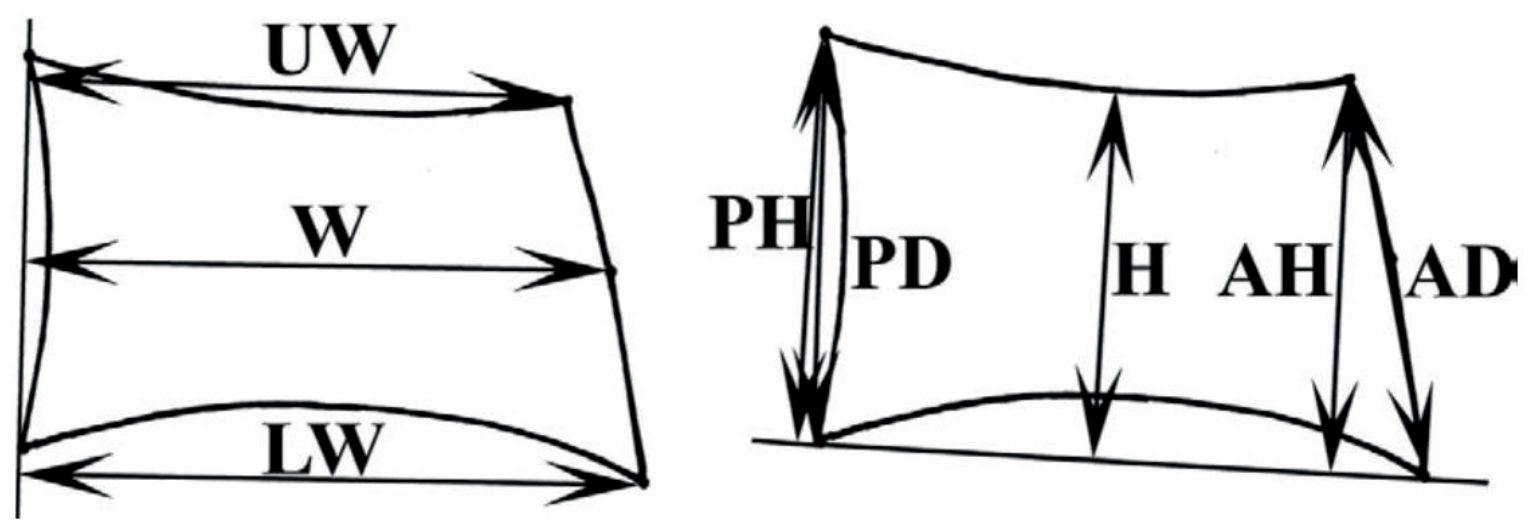

Figura 4. Líneas usadas en el análisis de los cuerpos vertebrales. UW, distancia horizontal de Cua a la conexión de Cup y Clp. W, distancia horizontal de Cam a la conexión de Cup y Clp. LW, distancia horizontal de Cla a la conexión de Cup y Clp. PH, distancia vertical de Cup a la conexión de Clp y Cla. $\mathrm{H}$, distancia vertical de Cum a la conexión de Clp y Cla. $\mathrm{AH}$, distancia vertical de Cua a la conexión de Clp y Cla. AD, distancia entre Cla y Cua.PD, distancia entre Clp y Cup. Fuente: Obtenido de Chen et al. ${ }^{21}$

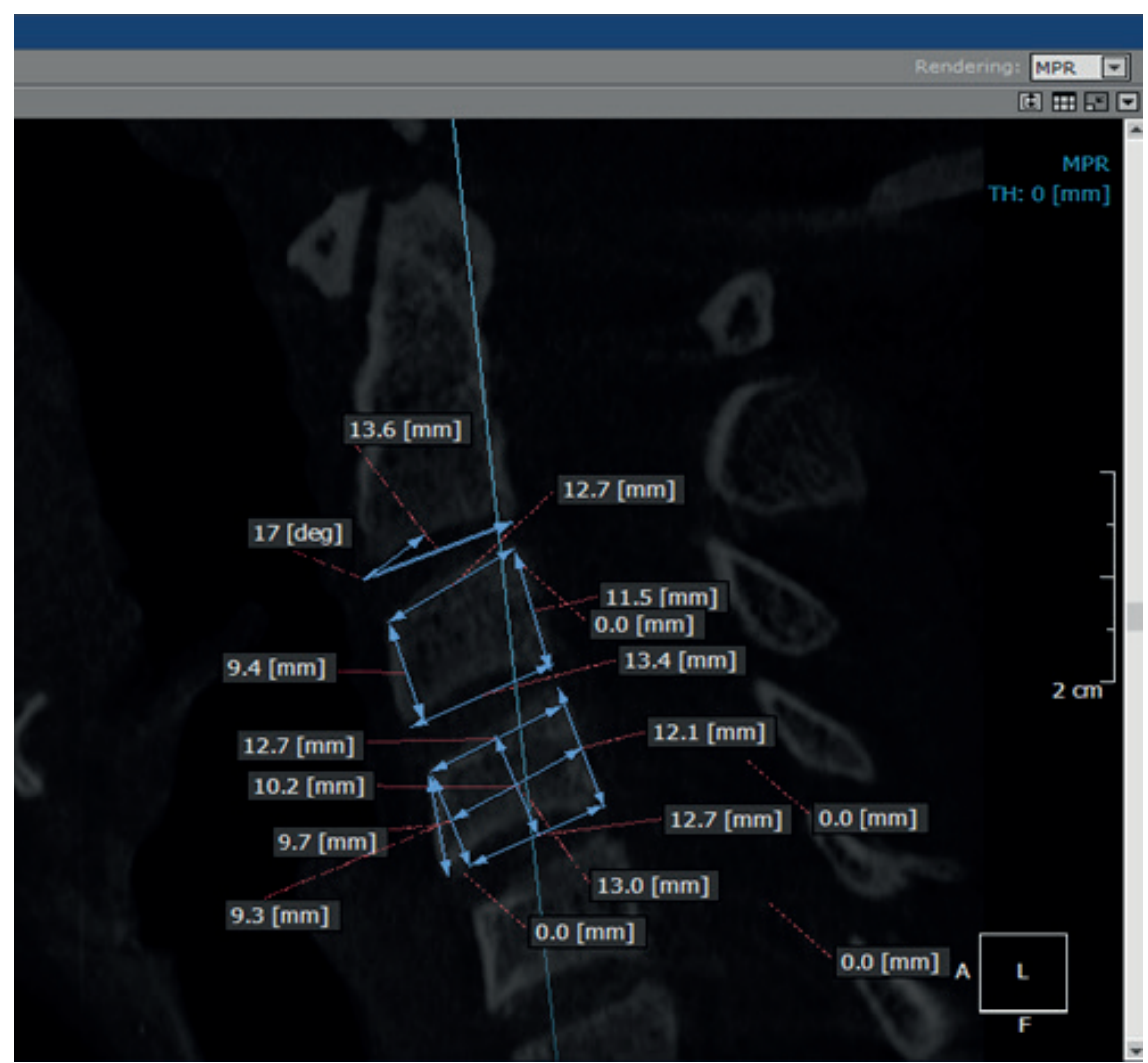

Figura 5. Análisis de los cuerpos vertebrales C2, C3 y C4 en vista sagital según Chen et al. ${ }^{21}$ Paciente de 12 años de edad, de sexo femenino. Medición angular de C2: @2, ángulo anterosuperior de C2d a C2p con la proyección de $\mathrm{C} 2 \mathrm{p}$ a C2a. Medición lineal de C3: AH3/PH3, proporción de AH3 a PH3. Medición lineal de C4: H4/W4, proporción de H4 a W4.

al método cuantitativo de maduración ósea en vértebras cervicales según Chen et al. ${ }^{21}$ (Figuras 3, 4 y 5).

Análisis de los cuerpos vertebrales de C2 a C4. De acuerdo al estudio de Chen $e t a l .{ }^{21}$ se tomaron tres mediciones: @2 ángulo antero superior de C2d a C2p con la proyección de $\mathrm{C} 2 \mathrm{p}$ a C2a, $\mathrm{AH} 3 / \mathrm{PH} 3$ y H4/W4.

Luego se procedió a ubicar los puntos que permitieron tomar las mediciones de cada vértebra, ya sea medición angular o mediciones lineales en la C2, C3 y C4 respec- tivamente otorgando a cada una de ellos una nomenclatura que se menciona a continuación: (Figuras 3 y 4 )

1. En el borde inferior de C2: el punto más posterior C2p, el más anterior C2a y el más profundo a nivel de la concavidad C2d.

2. En el borde superior de C3: el punto más posterior C3up, el más anterior C3ua. 
3. En el borde inferior de C3: el punto más posterior C3lp, el más anterior C3la y el más profundo a nivel de la concavidad C3d.

4. En el borde superior de C4: el punto más posterior C4up, el más anterior C4ua.

5. En el borde inferior de C4: el punto más posterior C4lp, el más anterior C4la y el más profundo a nivel de la concavidad C4d.

6. UW es la distancia desde Cua a la proyección de Cup y Clp

7. W distancia de Cam a la proyección entre Cup y Clp

8. LW distancia de Cla a la proyección de Cup y Clp

9. $\mathrm{PH}$ distancia de Cup a la proyección de Clp y Cla

10. H distancia entre Cum a la proyección de $\mathrm{Clp}$ a Cla

11. AH distancia de C3ua a la proyección de $\mathrm{Clp}$ a Cla

12. AD distancia entre Cla a Cua

13. PD distancia entre Clp a Cup

Luego que se obtuvieron las mediciones se procedió a realizar la siguiente ecuación sugerida por Chen et al. ${ }^{21}$ para determinar el período de maduración esquelética: CVMS $=-4.13+3.57^{*} \mathrm{H} 4 / \mathrm{W} 4+4.07^{*} \mathrm{AH} 3 /$ PH3+0.03*@2

Según el valor obtenido en la ecuación, Chen et al. ${ }^{21}$ se clasificó en cuatro estadios: QCVM I (periodo de aceleración) para valores de CVMS $<1,74$, QCVM II (periodo de alta velocidad) $1,74<\mathrm{CVMS}<2,62$, QCVM III (periodo de desaceleración) 2,62 $<$ CVMS $<3,52$, y QCVM IV (periodo de terminación) de CVMS > 3,52.

Los datos fueron analizados con el software estadístico Stata versión 12 . Se utilizó la estadística descriptiva para hallar las frecuencias y porcentajes. Previamente se determinó la concordancia interexaminador $(\mathrm{CCI}=0,91)$ y la concordancia intraexaminador $(\mathrm{CCI}=0,93)$.

\section{Resultados}

De las 14 CBCT evaluadas, siete fueron del sexo masculino (50\%). La distribución de la muestra en los diferentes estadios de maduración ósea según la ecuación de Chen et al. ${ }^{21}$ fue la siguiente: QCVM III que corresponde al periodo de desaceleración fue de $35,71 \%$, QCVM I que corresponde al periodo de aceleración fue de 28,57\%, QCVM II que corresponde al periodo de alta velocidad fue de $21,43 \%$ y QCVM IV que corresponde al periodo de terminación fue de 14,29\%. El mayor porcentaje se encontró en el QCVM III que corresponde al periodo de desaceleración (Tabla 1).

Tabla 1. Distribución de los períodos de maduración ósea propuesto por Chen et al ${ }^{21}$

\begin{tabular}{lll}
\multicolumn{1}{c}{ Estadio } & n & \% \\
\hline QCVM I & 4 & 28,5 \\
\hline QCVM II & 3 & 21,43 \\
\hline QCVM III & 5 & 35,71 \\
\hline
\end{tabular}

QCVM IV 14,29

Según sexo se observó que del total del sexo femenino, el $28,57 \%$ estuvieron en el estadio de maduración ósea QCVM II, el 28,57\% en QCVM III, el 28,57\% en QCVM IV y el 14,29\% en QCVM I. Se observó que del total del sexo masculino, el $42,86 \%$ estuvieron en el estadio de maduración ósea QCVM I, el 42,86\% en QCVM III, el 14,29\% en QCVM II y ninguno en QCVM IV. En QCVM II o periodo de alta velocidad se encontraron más niños del sexo femenino que masculino (Tabla 2). Al desglosar los resultados se apreció que, en este periodo, el promedio de edad alcanzado para las niñas fue de 11 años mientras que para los niños fue de 12 (Tabla 3 ).

Tabla 2. Distribución de los períodos de maduración ósea propuesto por Chen et al. ${ }^{21}$ según sexo ${ }^{21}$

\begin{tabular}{lcccc}
\hline \multirow{2}{*}{ Estadio } & \multicolumn{4}{c}{ Sexo } \\
\cline { 2 - 5 } & \multicolumn{2}{c}{ Femenino } & \multicolumn{2}{c}{ Masculino } \\
\cline { 2 - 5 } & $\mathbf{n}$ & $\mathbf{\%}$ & $\mathbf{n}$ & $\mathbf{\%}$ \\
\hline QCVM I & 1 & 14,29 & 3 & 42,86 \\
\hline QCVM II & 2 & 28,57 & 1 & 14,29 \\
\hline QCVM III & 2 & 28,57 & 3 & 42,86 \\
\hline QCVM IV & 2 & 28,57 & 0 & 0 \\
\hline
\end{tabular}

Tabla 3. Mediciones cuantitativas (angular y lineales) de la C2, C3 y C4 en CBCT según la ecuación y períodos de maduración ósea propuesto por Chen et al ${ }^{21}$

\begin{tabular}{cccccccc}
\hline Paciente Edad Sexo & @2 & AH3/PH3 & H4/W4 & Ecuación & $\begin{array}{c}\text { Estadio } \\
\text { QCVM }\end{array}$ \\
\hline 1 & 9 & M & 10,1 & 0,64 & 0,65 & 1,09 & QCVM I \\
\hline 2 & 9 & F & 4,6 & 0,63 & 0,85 & 1,6 & QCVM I \\
\hline 3 & 10 & M & 13,3 & 0,70 & 0,73 & 1,73 & QCVM I \\
\hline 4 & 10 & F & 20,1 & 0,73 & 0,88 & 2,6 & QCVM II \\
\hline 5 & 11 & M & 18,3 & 0,76 & 0,61 & 1,7 & QCVM I \\
\hline 6 & 11 & F & 20 & 0,89 & 0,76 & 2,8 & QCVM III \\
\hline 7 & 12 & M & 11,3 & 0,77 & 0,74 & 1,98 & QCVM II \\
\hline 8 & 12 & F & 17 & 0,82 & 0,78 & 2,51 & QCVM II \\
\hline 9 & 13 & M & 17,6 & 1,04 & 0,68 & 3,05 & QCVM III \\
\hline 10 & 13 & F & 12,6 & 0,93 & 1,38 & 4,97 & QCVM IV \\
\hline 11 & 14 & M & 16,3 & 0,87 & 0,93 & 3,22 & QCVM III \\
\hline 12 & 14 & F & 19 & 0,96 & 0,73 & 2,96 & QCVM III \\
\hline 13 & 15 & M & 17,3 & 0,78 & 0,96 & 2,99 & QCVM III \\
\hline 14 & 15 & F & 26,3 & 0,97 & 0,85 & 3,64 & QCVM IV \\
\hline
\end{tabular}

\section{Discusión}

El presente estudio evaluó el método cuantitativo de maduración ósea en vértebras cervicales propuesto por Chen et al. ${ }^{21}$ en CBCT. En relación a los resultados obtenidos se observó que usando este método se puede obtener precisión en establecer en qué periodo de maduración ósea se encuentra el niño a comparación de otros que evalúan solo cualitativamente las vértebras cervicales, como los de Lamparsky ${ }^{9}$, Hassel y Farman ${ }^{11}$ y Baccetti et al. ${ }^{12}$ y más aún al emplear la CBCT en este 
estudio, que es una herramienta de diagnóstico en Ortodoncia, hace que las medidas, tanto lineales como angulares de las vértebras $\mathrm{C} 2$, C3 y C4, obtenidas por medio del software anexado a dicha tomografía sean exactas para desarrollar la ecuación del método propuesto por Chen et al. ${ }^{21}$. De ese modo se obtuvo los resultados, con un método cuantitativo, de forma fácil, objetiva y precisa, así como otros estudios lo mencionan ${ }^{17,19,20 .}$

A pesar de los beneficios de un método cuantitativo obtenido con las mediciones de las vértebras C2, C3 y C4, Gray et al. ${ }^{22}$ cuestionaron mediante un análisis morfométrico de éstas vertebras (método cuantitativo analítico), que se pueda obtener el pico de crecimiento mandibular. Ellos midieron las vértebras con un método llamado Modelos de distribución de puntos (plantillas morfometricas), no muy usado en la actualidad ni con fines en investigación médica, además que su evaluación del pico de crecimiento mandibular fue con la longitud mandibular, la cual, su estudio no explica cómo fueron correlacionadas ambas.

Se observa que en la distribución de los períodos de maduración ósea propuesto por Chen et al. ${ }^{21}$ según sexo, el de mayor interés en Ortodoncia es el que el autor menciona como el periodo de alta velocidad (QCVM II), que vendría a ser el estadio de máxima aceleración de crecimiento, en donde se obtuvo que el promedio de edad alcanzado fue de 11 años para las niñas y de 12 para los niños, lo que demuestra que las niñas alcanzaron el pico de crecimiento mucho antes que los niños, resultados similares a otras investigaciones $14,19,23,25$.

Los resultados hallados en este estudio son importantes ya que es el primer estudio en Latinoamérica que evalúa la maduración ósea en vértebras cervicales con un método cuantitativo y que agrega una herramienta de diagnóstico, como es la CBCT, para hacerlo aún más preciso y objetivo a diferencia de otros métodos cuantitativos; cabe mencionar que Chen et al. ${ }^{21}$, lo realizaron en cefalogramas. Por consiguiente, la importancia de su aplicación clínica sería en el diagnóstico y en el tratamiento a realizar en Ortodoncia, ya que determinando el pico de crecimiento puberal se puede corregir problemas esqueletales. Aunque para evaluar un método cuantitativo en la determinación del pico de crecimiento mandibular, se sugiere que el estudio sea de tipo longitudinal como el de Chen et al. ${ }^{26}$ en el 2010, puesto que investigaciones de este tipo son de gran aporte para la evaluación del crecimiento facial. Son pocos los estudios longitudinales donde se evalúa la maduración ósea del individuo, así que se sugiere más estudios de este tipo para validar el método en una población peruana teniendo en cuenta otras variables intervinientes como talla, sexo y peso corporal, así como otros factores influyentes en el crecimiento (etnia, nutrición y nivel socioeconómico), como lo menciona Jiménez et al. ${ }^{5}$

Claro está que al tratarse de un estudio piloto, la principal limitación fue el número de muestra debido al acceso de las CBCT y al criterio de inclusión de homogeneidad de la muestra. Por ello, se sugiere también ampliar el número de muestra para comparar este método tan- to en CBCT como en radiografías cefalométricas, con otros métodos como el de mano muñeca y así validar y determinar un método cuantitativo aplicable y válido a nuestra población.

Se concluye que el QCVM brindó un enfoque cuantitativo específico, preciso y objetivo para identificar las etapas de maduración ósea de los niños y puede tener aplicación clínica para determinar el pico de crecimiento puberal y así corregir problemas esqueletales de la mandíbula.

El estadio de máxima aceleración de crecimiento, considerado el de mayor interés en Ortodoncia, en este método cuantitativo fue el QCVM II, en donde se obtuvo, que el promedio de edad alcanzado por las niñas fue de 11 años y para los niños los 12 ańos.

Las imágenes obtenidas por la CBCT permitieron una medición exacta de las líneas y ángulo que se emplearon en el QCVM

\section{Agradecimientos}

Especial agradecimiento al CD. Esp. Edgar Armando Noli Lazo por su apoyo en la enseñanza del uso de la CBCT.

\section{Referencias bibilográficas}

1. San Román P, Palma JC, Oteo MD, Nevado E. Skeletal maturation determined by cervical vertebrae development. Eur J Orthod. 2002; 24(3):303-11.

2. Flores-Mir C, Burgess CA, Champney M, Jensen RJ, Pitcher MR, Major PW. Correlation of skeletal maturation stages determined by cervical vertebrae and handwrist evaluations. Angle Orthod. 2006;76:1-5. DOI: 10.1043/0003-3219(2006)076[0001:COSMSD]2.0. $\mathrm{CO} ; 2$

3. Fishman LS. Maturational patterns and prediction during adolescence. Angle Orthod. 1987;57:178-93. DOI: 10.1043/0003-3219(1987)057<0178:MPAPDA>2.0. $\mathrm{CO} ; 2$

4. Baccetti T, Franchi L, McNamara JA Jr. The Cervical Vertebral Maturation (CVM) Method for the Assessment of Optimal Treatment Timing in Dentofacial Orthopedics. Semin Orthod. 2005;11(3):119-29. DOI: 10.1053/j.sodo.2005.04.005

5. Jiménez ID, Villegas LF, Álvarez LG. Crecimiento facial vertical en 44 mestizos colombianos sin tratamiento desde los 6 hasta los 25 ańos Grupo CES-LPH. Rev CES Odont. 2011;24(2):9-33.

6. Fishman LS. Radiographic evaluation of skeletal maturation: A clinically oriented method based on handwrist films. Angle Orthod. 1982;52:88-112. DOI: 10.1043/0003-3219(1982)052<0088:REOSM>2.0. $\mathrm{CO} ; 2$

7. Perinetti G, Contardo L. Reliability of Growth Indicators and Efficiency of Functional Treatment for Skeletal Class II Malocclusion: Current Evidence and Controversies. Biomed Res Int. 2017; 2017:1367691. DOI: $10.1155 / 2017 / 1367691$

8. Cericato GO, Bittencourt MAV, Paranhos LR Validity of the assessment method of skeletal maturation by cer- 
vical vertebrae: a systematic review and meta-analysis. Dentomaxillofac Radiol. 2015;44(4):20140270. DOI: $10.1259 / \mathrm{dmfr} .20140270$

9. Lamparski DG. Skeletal age assessment utilizing cervical vertebrae. Am J Orthod Dentofacial Orthop. 1975;67(4):458-9. DOI: http://dx.doi.org/10.1016/0002-9416(75)90038-X

10. O’Reilly MT, Yanniello GJ. Mandibular growth changes and maturation of cervical vertebra a longitudinal cephalometric study. Angle Orthod. 1988;58:179-84. DOI: 10.1043/0003-3219(1988)058<0179:MGCAMO>2.0. $\mathrm{CO} ; 2$

11. Hassel B, Farman A. Skeletal maturation evaluation using cervical vertebrae. Am J Orthod Dentofacial Orthop 1995;107:58-66.

12. Baccetti T, Franchi L, Mc Namara JA Jr. An improved version of the cervical vertebral maturation (CVM) method for the assessment of mandibular growth. Angle Orthod. 2002;72(4):316-23. DOI: 10.1043/0003-3219(2002)072<0316:AIVOTC>2.0 $\mathrm{CO} ; 2$

13. Wong RK, Alkhal HA, Rabie AB. Use of cervical vertebral maturation to determine skeletal age. Am J Orthod Dentofacial Orthop 2009;136:484.e1-6. DOI: 10.1016/j.ajodo.2007.08.033

14. Shim JJ, Heo G, Lagravére MO. Assessment of skeletal maturation based on cervical vertebrae in CBCT. Int Orthod. 2012;10(4):351-62. DOI: 10.1016/j.ortho.2012.09.005

15. Chatzigianni A, Halazonetis DJ. Geometric morphometric evaluation of cervical vertebrae shape and its relationship to skeletal maturation. Am J Orthod Dentofacial Orthop. 2009;136(4):481.e1-9. DOI: 10.1016/j. ajodo.2009.04.017.

16. Gabriel DB, Southard KA, Qian F, Marshall SD, Franciscus RG, Southard TE. Cervical vertebrae maturation method: poor reproducibility. Am J Orthod Dentofacial Orthop. 2009;136(4):478.e1-7. DOI: 10.1016/j.ajodo.2007.08.028

17. Mito T, Sato K, Mitani H. Cervical vertebral bone age in girls. Am J Orthod Dentofacial Orthop 2002;122:380-5.

18. Chen F, Terada K, Hanada K. A new method of predicting mandibular length increment on the basis of cervical vertebrae. Angle Orthod. 2004;74:630-4. DOI:
10.1043/0003-3219(2004)074<0630:ANMOPM>2.0. $\mathrm{CO} ; 2$

19. Santiago RC, Cunha AR, Júnior GC, Fernandes N, Campos MJ, Costa LF, et al. New software for cervical vertebral geometry assessment and its relationship to skeletal maturation - a pilot study. Dentomaxillofac Radiol. 2014;43(2):20130238. DOI: 10.1259/dmfr.20130238.

20. Byun BR, Kim YI, Yamaguchi T, Maki K, Son WS. Quantitative assessment of cervical vertebral maturation using Cone Beam Computed Tomography in Korean girls. Comput Math Methods Med. 2015;19(8):213340. DOI: $10.1155 / 2015 / 405912$

21. Chen LL, Xu TM, Jiang JH, Zhang XZ, Lin JX. Quantitative cervical vertebral maturation assessment in adolescents with normal occlusion: a mixed longitudinal study. Orthod Dentofacial Orthop. 2008;134(6):720. DOI: 10.1016/j.ajodo.2008.03.014

22. Gray S, Bennani H, Kieser JA, Farella M. Morphometric analysis of cervical vertebrae in relation to mandibular growth. Am J Orthod Dentofacial Orthop. 2016;149(1):92-8. DOI: 10.1016/j.ajodo.2015.06.028.

23. Joshi V, Yamaguchi T, Matsuda Y, Kaneko N, Maki K, Okano T. Skeletal maturity Med Oral Pathol Oral Radiol. 2012;113:841-9. DOI: 10.1016/j.oooo.2011.11.018

24. Swennen GR, Schutyser F. Three-dimensional cephalometry: Spiral multi-slice vs cone-beam computed tomography. Orthod Dentofacial Orthop. 2005;130(3):4106. DOI: $10.1016 /$ j.ajodo.2005.11.035

25. Byun BR, Kim YI, Yamaguchi T, Maki K, Ko CC, Hwang DS, et al. . Quantitative skeletal maturation estimation using cone- beam tomography - generated cervical vertebral images: a pilot study in 5-to 18-year old Japanese children. Clin Oral Invest. 2015;19(8):2133-40. DOI: $10.1007 / s 00784-015-1415-6$.

26. Chen L, Liu J, Xu T, Lin J. Longitudinal study of relative growth rates of the maxilla and the mandible according to quantitative cervical vertebral maturation. Am J Orthod Dentofacial Orthop. 2010;137(6):736.e1-8. DOI: 10.1016/j.ajodo.2009.12.022. 
